\title{
Extend the power of cellular models
}

\author{
Peter Nick
}

Received: 30 November 2011 / Accepted: 30 November 2011 /Published online: 11 December 2011

(C) Springer-Verlag 2011

Model organisms have advanced biology tremendously because they are amenable to genetic analysis and manipulation. However, the power of model organisms depends on the transferability of the results. For instance, the model plant Arabidopsis thaliana is not intensively studied by its own virtue, but because the results can be projected to other plants of interest, including economically important crop plants. In many cases, it is this transfer that limits the power of a model. Two strategies have been employed to overcome this limitation: either, (a) reconstitute transferability by refined tools of detection or methods of manipulation or (b) introduce new model organisms that are closer to the application of interest. The current issue assembles examples for both approaches:

...extend the power of existing models

It is hard to believe that the tiny weed $A$. thaliana might serve as model to understand the growth of mighty trees, but the technical advance by Mazur and Kurczynska (2011) in the current issue is exactly aiming to render thale cress a suitable model for secondary growth of trees. Secondary growth had already been studied in hypocotyls of $A$. thaliana (Chaffey et al. 2002), but was found to lack both secondary rays and intrusive cambial cell growth as central features of secondary thickening of trees (Barlow et al. 2010). By subjecting decapitated flower stems to mechanical load, Mazur and Kurczynska (2011) could close this gap and induce secondary growth including secondary rays and intrusive cambial growth thus exactly mimicking the situation in a 'real' tree. This technical advancement opens wood

Handling Editor: Peter Nick

P. Nick $(\bowtie)$

Karlsruhe, Germany

e-mail: peter.nick@bio.uka.de research for all the powerful mutant and molecular approaches available in the thale cress model.

To investigate the function of cancer proteins in a plant system, sounds odd at first sight. However, since plant development is more robust and flexible, in many cases, specific phenotypes can be observed and analysed even for genes whose manipulation in the animal system would result in mere lethality obscuring possible functions. The work by Polit et al. (2011) in the current issue investigates the cell cycle-dependent phosphorylation of plant homologues for the retinoblastoma tumour suppressor protein $(\mathrm{pRb})$ controlling the G1-to-S-transition in the mammalian cell cycle and therefore representing an important player in the genesis and therapy of human cancers. By using a specific antibody recognising phosphorylations in specific serines of the so-called $\mathrm{C}$-domain of $\mathrm{pRb}$ (essential to transmit the signal to downstream effectors) in root meristems of Vicia faba, a welldefined cellular model for cell division in a tissue context, they can demonstrate that the C-domain is transiently phosphorylated at the G1-to-S-transition. This suggests that this aspect of cell cycle regulation is evolutionarily conserved, which is surprising given the fact that plant $\mathrm{pRb}$-homologues are much smaller than their animal counterparts.

.... and search for new ones

The central concept of the model approach is to search for generalisations. However, many essentials of biology are not general, but specific - specificity has been a central driving force of evolution and governs, for instance, most interactions between organisms. Here, the strategy to use few model organisms and to extend their value by sophisticated evaluation rapidly runs into a dead end. Organismic interactions - including the medically or agronomically important interactions with pathogens - can only be studied in appropriate pairs of host and pathogen. Here, transferability 
gains priority over genetic accessibility. In their invited review, Beakes et al. (2011) illustrate the evolutionary phylogeny of oomycetes, a group of protists, erroneously termed as 'fungi'. Most oomycetes have adopted a lifestyle as biotrophic pathogens that are able to reprogramme the immune response of their respective hosts for their specific needs. The specificity of this interaction has resulted in a tremendous radiation sometimes resulting even in host species-specific pathogen species. This example nicely illustrates the limitations posed to transferability of model organisms in plant pathology and the recruit non-canonical model organisms. As an illustration for this conclusion, Song et al. (2011) in the current issue use information derived from the model system barley to understand the interaction between the economically important wheat pathogen Puccinia striiformis and wheat. Wheat is anything else than an ideal model organism-a hexaploid complex of three different genomes, hard to be transformed and so far not even fully sequenced does not qualify this species as a good model. However, wheat is the economically most relevant staple food and it is the host for $P$. striiformis. The authors circumvent the difficulties of wheat as genetic system by fishing interesting gene candidates using subtractive hybridisation of infected wheat leaves and identifying and cloning interesting candidates based on their homology with sequences obtained from the barley system. By this approach, they clone a wheat homologue of the actin-binding protein profilin that is upregulated during incompatible, but not during compatible, interaction consistent with previous observations that profilin as well as Rop GTPases accumulate at infection sites of plant cells (Schütz et al. 2006). Using wheat as cell biological model, they can eventually verify their hypothesis that actin remodelling is relevant for the outcome of host-pathogen interaction by pharmacological manipulation of actin.

\section{References}

Barlow PW, Mikulecky S, Střeštik J (2010) Tree-stem diameter fluctuates with the lunar tides and perhaps with geomagnetic activity. Protoplasma 247:25-43

Beakes GW, Glockling SL, Sekimoto S (2011) The evolutionary phylogeny of the oomycete "fungi". Protoplasma doi:10.1007/ s00709-011-0269-2

Chaffey N, Cholewa E, Regan S, Sundberg B (2002) Secondary xylem development in Arabidopsis: a model for wood formation. Physiol Plant 114:594-600

Mazur E, Kurczynska EU (2011) Rays, intrusive growth and storied cambium in the inflorescence stems of Arabidopsis thaliana (L.) Heynh. Protoplasma doi:10.1007/s00709-011-0266-5

Polit JT, Kaźmierczak A, Walczak-Drzewiecka A (2011) Cell cycledependent phosphorylation of $\mathrm{pRb}$-like protein in root meristem cells of Vicia faba. Protoplasma doi:10.1007/s00709-011-0272-7

Schütz I, Gus-Mayer S, Schmelzer E (2006) Profilin and Rop GTPases are localized at infection sites of plant cells. Protoplasma 227:229235

Song X, Ma Q, Hao XY, Li HL (2011) Roles of the actin cytoskeleton and an actin-binding protein in wheat resistance against Puccinia striiformis f. sp. tritici. Protoplasma doi:10.1007/s00709-0110265-6 